\title{
AFM, SEPM AND CONTACT ANGLE SURFACE ANALYSIS ON LOW DENSITY POLYETHYLENE GRAFTED WITH MALEIC ANHYDRIDE
}

\author{
M. F.Porto ${ }^{1}$, M. H. Kunita ${ }^{1}$, E. Radovanovic ${ }^{1}$, M.C.Gonçalves ${ }^{2}$, E.C. Muniz ${ }^{1}$, and A. F. Rubira ${ }^{1 *}$ \\ ${ }^{1}$ Grupo de Materiais Poliméricos e Compósitos - Departamento de Química \\ Universidade Estadual de Maringá - Av. Colombo 5790, 87020-970 - Maringá - Paraná - Brazil, \\ ${ }^{2}$ Instituto de Química - UNICAMP, C. Postal 6154, 13083-970 Campinas-SP-Brazil
}

The modification of polymer surfaces has received a great attention during the last years, due to the search for new materials. Polymers of great commercial use such as polyethylene, polypropylene and polystyrene have had their properties modified, especially the surface properties. However, many researchers have recently altered the properties of polymers via grafting reactions. In a grafted polymer one or more types of blocks are connected to the main polymer as side chains, that are constituted or configured differently from the main polymer. The surface modification of lowdensity polyethylene (LDPE) has been made in our group by the grafting of maleic anhydride, in order to accomplish subsequent reactions, generating reactive functional groups on the LDPE surface. In this work, the surface of the polyethylene submitted to a grafting with maleic anhydride followed by hydrolysis, chlorination, amination and protonation reactions was analyzed by Atomic Force Microscopy (AFM) and water contact angle. Low-density polyethylene in pellet was pressed at $170^{\circ} \mathrm{C}$ and 2 ton. of pressure to obtain films of average thickness of $110 \mu \mathrm{m}$. The sample was immersed in a solution of maleic anhydride $(4.0 \mathrm{~g})$ in acetic anhydride $(80 \mathrm{~mL})$, using benzoyl peroxide $(0.22 \mathrm{~g})$ as radicals initiator, heated at $100^{\circ} \mathrm{C}$, under agitation, for 6 hours. After, the sample was hydrolyzed at $100^{\circ} \mathrm{C}$ for 5 hours, followed by immersion in a solution of $2 \%$ in volume of antimony pentachloride in carbon tetrachloride, for 24 hours at $35^{\circ} \mathrm{C}$. The next immersions were in i) triethanolamine and ii) phosphoric acid, also under agitation, both for 24 hours at room temperature. The contact angle analyses were performed in a TANTEC equipment, CAM-Micro model. AFM and SEPM images of modified PE were acquired using a Topometrix Discoverer instrument. SEPM in this instrument uses the standard non-contact AFM set-up, but with some modifications that allow that a tip covered with Pt to detect electric potential gradients throughout the scanned area. Besides, the obtainment of the $\mathrm{Z}$ scale in the AFM images enables the analysis of several important characteristics of the materials surfaces, relative to the roughness of the material, and has been used in the studies of the changes that occur on the surface of polymeric materials due to the treatments. A roughness parameter directly calculated by the software of the microscope was used in these analyses: the average roughness ( $\mathrm{Ra})$ which corresponds to the arithmetic mean of the deviations in height from the image mean value. In the investigation on the modification of polyethylene via grafting, the surface roughness can be an important factor of process accompanying. In the micrograph of virgin polymer surface, as well as the micrograph of the hydrolyzed surface showed in Figure 1 (scanning area of 2,5 x 2,5 $\mu \mathrm{m}$ ), it is possible to observe that the mountain-shaped structures with global peak in the virgin polyethylene have become peaky in the hydrolysis step. This is probably due to the insertion of maleic anhydride following by hydrolysis. The Ra values for the virgin LDPE and modified LDPE films calculated from a series of images with $10 x 10 \mu \mathrm{m}$ are presented in Figure 2A. The Ra values obtained in a same sample in different areas varied between $6 \%$ and $25 \%$ from the average values. In spite of the great values of average deviation, it is possible to conclude that the polyethylene surface becomes less rough with the hydrolysis process, going back to values close to the virgin polyethylene in the following steps. This fact can probably be associated 
to the formation of a less hydrophobic structure with the addition of acid groups, in comparison to the other samples. This fact is confirmed by the contact angle analysis showed in Figure 2B. Besides, in SEPM micrographs (Fig. 3) it is possible to see that occurred an accumulation of charge correlated with the topography features in micrographs related to hydrolyzed LDPE (Fig. 3C and 3D) in contrast with no charge differences in virgin LDPE (Fig. 3A and 3B). Most often, the elevations in hydrolyzed polymer image contain an excess of positive electric charges (or a deficiency of negative charges), relative to surface depressions.

Acknowledgements: CNPq, Capes, Fapesp, Fundação Araucária, Unicamp and UEM.

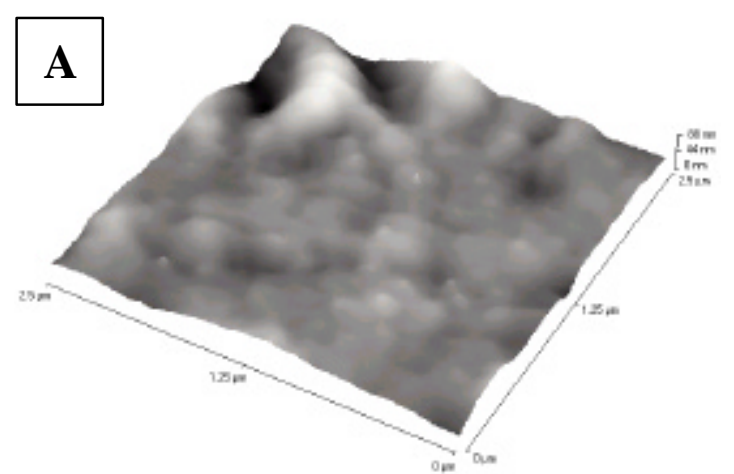

Figure 1 - AFM images of virgin LDPE (A), followed by grafting (B), hydrolysis (C), amination(D) and protonation (E).

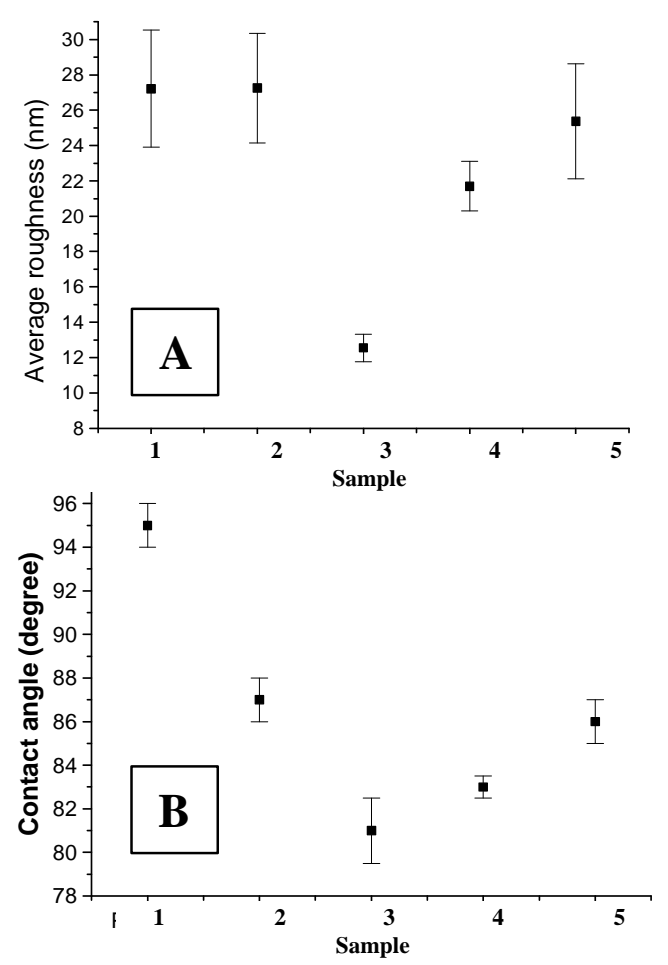

Figure 2 - (A)Ra values calculated from $10 \times 10 \mu \mathrm{m}$ images and (B) Average contact angle for the LDPE. 1) virgin; 2) grafted; 3) hydrolyzed; 4) aminated and 5) protonated.
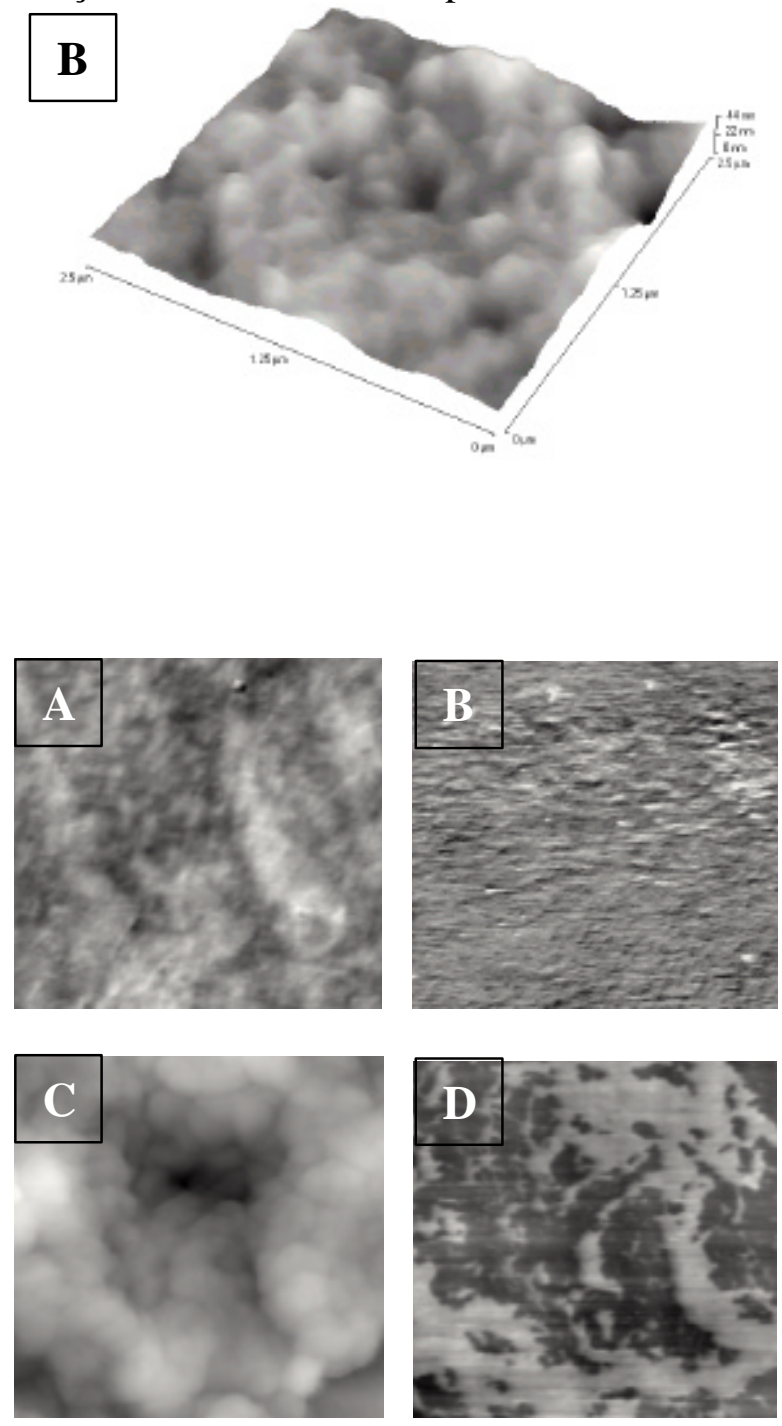

Figure 3 - AFM $(7 \times 7 \mu \mathrm{m})$ topography images (A and $C$ ) and respective SEPM images (B and D) of virgin LDPE (A and B) and hydrolyzed LDPE (C and D). 